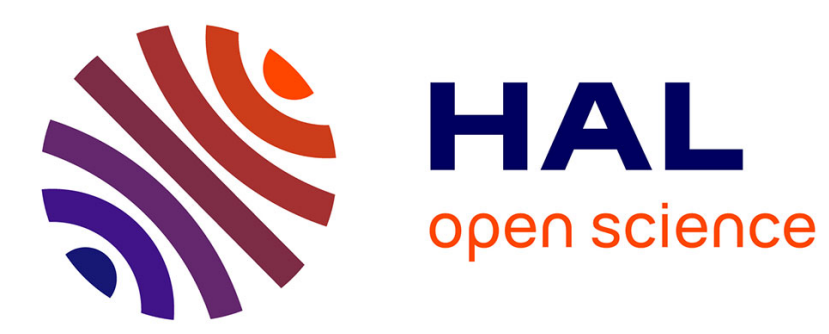

\title{
Usage de la morphométrie dans la révélation des préférences de mobilité. Application aux cheminements piétons
}

Arnaud Piombini, Thomas Leduc, Philippe Woloszyn

\section{To cite this version:}

Arnaud Piombini, Thomas Leduc, Philippe Woloszyn. Usage de la morphométrie dans la révélation des préférences de mobilité. Application aux cheminements piétons. Revue Internationale de Géomatique, 2014, 24 (1), pp.101-130. 10.3166/rig.24.101-130 . hal-01345703

\section{HAL Id: hal-01345703 \\ https://hal.science/hal-01345703}

Submitted on 15 Jul 2016

HAL is a multi-disciplinary open access archive for the deposit and dissemination of scientific research documents, whether they are published or not. The documents may come from teaching and research institutions in France or abroad, or from public or private research centers.
L'archive ouverte pluridisciplinaire HAL, est destinée au dépôt et à la diffusion de documents scientifiques de niveau recherche, publiés ou non, émanant des établissements d'enseignement et de recherche français ou étrangers, des laboratoires publics ou privés. 


\title{
Usage de la morphométrie dans la révélation des préférences de mobilité
}

\section{Application aux cheminements piétons}

\author{
Arnaud Piombini' ${ }^{1}$, Thomas Leduc ${ }^{2}$, Philippe Woloszyn ${ }^{3}$ \\ 1. Laboratoire Image, Ville, Environnement - ERL 7230 CNRS, Université de \\ Strasbourg \\ 3 rue de l'Argonne, 67083 Strasbourg, France \\ arnaud.piombini@live-cnrs.unistra.fr
}

2 CERMA - UMR 1563 CNRS, LUNAM Université, École nationale supérieure d'architecture de Nantes 6 quai François Mitterrand, BP16202, 44262 Nantes Cedex 2, France thomas.leduc@cerma.archi.fr

\author{
2 ESO - UMR 6590 CNRS, Université Rennes 2 \\ Maison de la Recherche en Sciences Sociales, 35043 rennes, France \\ philippe.woloszyn@univ-rennes2.fr
}

\begin{abstract}
RESUME. La compréhension des comportements de mobilité pédestre repose habituellement sur des analyses agrégées établissant une relation entre des entités spatiales et les flux de mobilité qui les traversent. Cette approche est notamment utilisée dans le but d'analyser le rôle de la morphologie bâtie sur les comportements pédestres. Pour aller plus loin, nous proposons une analyse désagrégée, issue des modèles de choix discret, et ciblée sur les comportements de chaque piéton. Dans cette optique, nous confrontons divers indices morphométriques à des choix d'itinéraires relevés lors d'une enquête à Strasbourg. Les résultats obtenus sont encourageants et montrent que cette méthode devrait être plus généralement utilisée pour mettre en évidence le rôle de la morphologie bâtie sur les pratiques de déplacement pédestres.

ABSTRACT. The understanding of pedestrian mobility behaviors often relies on aggregated analyses. These analyses correlate spatial entities with the mobility streams that cross them. These types of approach are in particular developed to assess the impact of built morphology on pedestrian behavior. To go a bit further, we propose an unaggregated analysis, based on discrete choice models, and targeted on each pedestrian behavior. Thus, we check several morphometric indices with some route choices obtained during a survey in Strasbourg city. The result gives us confidence and shows that the method we present should be broadly distributed to exhibit the impact of built morphology on pedestrian mobility.
\end{abstract}

Revue internationale de Géomatique $-\mathrm{n}^{\circ}$ 
Morphométrie et préférences de mobilité 2

MOTS-CLES: Mobilité pédestre, morphologie urbaine, analyse morphométrique, comportements de mobilité

KEYWORDS: Walking, built morphology, morphometry analysis, mobility behavior

DOI:10.3199/JESA.45.1-n @ Lavoisier 2012 


\section{Introduction}

Pour identifier les liens entre mobilité et espaces urbains, les approches privilégiées s'appuient généralement sur les préconisations de la charte d'Aalborg. La mobilité intra-urbaine est ainsi croisée à des mesures portant sur la densité de la population, des emplois, la diversité fonctionnelle, les caractéristiques du réseau viaire (Cervero et Kockelman, 1997). En revanche, alors que de nombreuses mesures morphométriques appliquées au tissu urbain à une échelle locale sont utilisées, en architecture notamment, la confrontation aux pratiques de mobilité reste peu mise en œuvre. Pourtant, il semble que l'on connaisse peu l'incidence de la morphologie bâtie sur les pratiques de déplacement, notamment pédestres (Breheny, 1992).

Dans cet article, nous présentons un ensemble de mesures reposant sur des principes de visibilité, par nature importante dans le registre de la mobilité urbaine et que nous assimilons en partie à la notion d'ambiances urbaines. Les ambiances architecturales et urbaines reposent sur l'ensemble des sens humains et correspondent à l'ensemble des phénomènes physiques de l'environnement construit et ce que les sens en perçoivent (Augoyard, 1979). Elles sont à la croisée des éléments physiques et quantifiables de la ville et de l'expérience personnelle et collective des citadins (Péneau, 2000). Les villes n'existent que par le prisme de la perception de leurs habitants (Lynch, 1969) et les ambiances se trouvent à l'interface de la structure spatiale «objective » de la ville et des pratiques individuelles plus subjectives (Santos, 1997).

Dans cette optique, notre objectif est de montrer qu'il est possible d'identifier certaines préférences ambiantales des citadins par une méthode d'analyse des itinéraires pédestres. Par rapport aux autres modes de transport, la marche à pied implique une immersion forte dans l'espace du mouvement. Elle induit un rapport très sensible à l'environnement urbain (Amar, 1993) c'est-à-dire une interaction forte entre l'individu et l'espace. Cette interaction repose sur des besoins que l'environnement urbain doit satisfaire pour que le piéton se sente à l'aise et ne renonce pas à ce mode de déplacement. Ceux-ci peuvent être hiérarchisés : les ambiances urbaines n'interviennent qu'après certains besoins comme les facilités de déplacements, la sécurité que le réseau viaire et les espaces publics procurent aux piétons (Alfonzo, 2005) ${ }^{1}$. Pour autant, il ne faut pas négliger les éléments d'ambiances qui jouent un rôle fondamental dans le maintien des pratiques pédestres et dans la qualité des espaces publics.

A cette fin, nous considérons que le piéton est un «capteur multi-sensoriel » des ambiances urbaines. Les choix d'usages qu'il va mettre en œuvre témoignent des liens qui l'unissent aux espaces urbains et permettent de révéler les significations sensibles des lieux ce qui leur confère un grand intérêt (Thomas, 2004). L'objectif consiste alors à mettre en évidence les facteurs influençant les micro-mobilités

1. La pyramide des besoins pédestres proposée par Alfonzo (2005) s'inspire de la hiérarchie des besoins humains de Maslow 
piétonnes (Besse, 2010) pour comprendre comment l'on passe d'un espace construit à un espace signifiant, pratiqué. Cette approche renvoie à la sémiotique de l'espace, c'est-à-dire à l'étude des signes que produisent les lieux par leurs propriétés élémentaires et à leur signification pour les individus.

Ainsi, au sein d'un espace «ressource» dont les ambiances sont, par nature, hétérogènes et qui présente de multiples possibilités d'usage, les choix de déplacement des piétons peuvent être utilement analysés. Sur la base d'observations individuelles on peut aboutir à une compréhension collective des pratiques urbaines. Ce point est essentiel car il permet d'introduire une méthode d'analyse des choix issue de l'économie qui peut être utilisée dans une approche spatiale (Foltête et Piombini, 2010). Ces modèles de choix discrets permettent d'analyser la prise de décision à un niveau individuel pour ensuite proposer des explications valables pour un échantillon donné. Cette approche désagrégée est intéressante car la plupart des travaux liant morphologie bâtie et mobilité reposent sur des analyses agrégées basées sur les relations entre les entités spatiales (tronçon de rue, ligne axiale, isovist ${ }^{2}$ ) et les flux de mobilité qui les parcourent alors que, dans le cas présent, nous centrons notre étude sur les comportements de chaque individu. La méthode proposée consiste en une valuation multiple, sur des critères morphométriques, de l'ensemble des tronçons de rues ${ }^{3}$ constitutifs de notre terrain d'étude. Un relevé des parcours pédestres par une enquête de terrain permet ensuite d'utiliser un modèle d'analyse des choix susceptible de mettre en évidence les indices morphométriques ayant une influence sur les choix d'itinéraires.

\section{Eléments méthodologiques}

\subsection{Principes de l'analyse morphométrique}

Dans la liste des nombreux facteurs influençant les micro-mobilités piétonnes, nous avons délibérément choisi d'analyser ceux liés à la perception géométrique et topologique de la «boite urbaine ». Les données permettant d'étudier ces paramètres sont en effet les plus faciles à acquérir (les bases de données vecteur de référence, de précision métrique, contiennent les éléments paysagers correspondant), elles assurent aussi, par leur couverture systématique du territoire national, une reproductibilité de la méthode. Nous ne traiterons donc pas ici des influences liées à la texturation ou à la couleur des éléments de paysage, à la typicité comme à la cohérence du site, à sa micro-climatique, aux effets d'éblouissements et de contraste, aux affluences d'usage, etc.

La démarche que nous proposons ne vise pas à produire une analyse morphologique complète de la zone d'intérêt. En effet, une telle analyse repose traditionnellement sur trois évaluations différentes du tissu urbain. La première

2. L'isovist (Benedikt, 1979) est un polygone formé de l'ensemble des portions de l'espace visibles depuis un lieu d'observation donné.

3. Le tronçon constitue l'entité de base du réseau viaire, au sens circulatoire du terme. 
consiste en une quantification morphométrique des empreintes de bâti, c'est-à-dire en une évaluation des propriétés géométriques de chaque objet construit considéré isolément. La seconde consiste en une analyse des configurations spatiales; il s'agit en l'occurrence d'étudier les proximités, mitoyennetés, continuités, etc. des empreintes de bâtiments. La troisième et dernière a pour but de préciser la nature de la distribution spatiale du bâti mise en œuvre (étude de densité, éventuelles régularités, etc.). Or, ces trois démarches concourantes présupposent, pour être menées à bien, un point de vue global, extérieur, zénithal à la zone d'étude. Un piéton en situation de mobilité ne peut, en règle générale, avoir de point de vue que local, immergé, tangentiel.

Notre approche se résume donc à quantifier l'espace environnant du piéton en chaque point du réseau. Il existe dans la littérature un certain nombre de tentatives de qualification d'espaces ouverts. Une démarche reposant sur l'ontologie plenum ((Couclelis, 1992) - par opposition à la conception atomiste de l'espace bâti), approche orientée champs, nous semble la plus pertinente ${ }^{4}$. Elle borne en effet l'espace ouvert environnant à l'horizon immédiat du piéton en lui opposant l'ensemble des arêtes opaques de la couche bâtie d'une part et les arêtes occlusives dues aux effets de masque $\mathrm{d}^{5}$ 'autre part. Dans cette approche, que l'on peut qualifier de simple « lancer de rayons » dans le plan de visibilité, les surfaces construites sont idéalement absorbantes et les réflexions supprimées. L'espace ouvert de déambulation du piéton est ainsi capté, délimité et de facto quantifiable et quantifié.

Dans ce registre de l'analyse visuelle des formes des espaces ouverts immédiatement environnants, sont recensées des approches empiriques telles que l'étude de séquences picturales proposée par Panerai et al. (1999). Dans cette approche, une grammaire de plans et séquences est proposée qui peut-être mise en relation avec la morphologie des lieux.

En complément de ces approches empiriques, diverses méthodes d'analyse quantitative des variations morphologiques des espaces ouverts au cours de la déambulation ont été définies. Nous éliminerons les approches dites de partitionnement convexe (Peponis et al., 1997), les lignes et cartes axiales de la space syntax (Hillier et Hanson, 1984) ou les graphes de visibilité (Turner et al., 2001). En effet, ces trois méthodes, bien que construites à partir de critères liés à la perception d'un sujet en situation de mobilité, procèdent globalement d'une approche

4. Selon Couclelis (1992) rapporté par Sarradin (2004), deux approches ontologiques différentes sont envisageables pour caractériser les espaces urbains. Dans la conception atomiste, l'espace est assimilé à un ensemble d'objets "solides" spatialement bien circonscrits. A contrario, l'approche plenum de l'espace urbain, consiste à appréhender l'espace comme un champ continu à l'image d'un champ magnétique. Dans la conception plenum, l'espace résulte d'une combinaison de facteurs tels que la répartition des masses construites par exemple. Toute modification du bâti en un point de l'espace urbain influe, directement ou non, sur les espaces ouverts immédiatement environnants.

5. En effet, l'enveloppe de l'espace immédiatement perceptible par un piéton est constituée soit de façades bâties, soit d'un ensemble de faces immatérielles résultant d'effets d'obturation provoqués par les façades qui s'intercalent. 
plus intégrée ou plus agrégée de l'espace d'étude et ne semblent pas être adaptées à la qualification d'un point de vue immergé. Par ailleurs, comme constaté dans Sarradin (2004), les techniques de partitionnement convexe de l'espace ouvert comme le découpage en s- voire e- partitions $^{6}$, si elles semblent adaptées aux espaces architecturaux, sont difficilement transposables aux espaces urbains pour lesquels le partitionnement devient rapidement inintelligible car trop complexe. Enfin, en ce qui concerne les approches axiales de la space syntax, nous souscrivons aux réserves évoquées dans Ratti (2004).

Les cartes de vue du ciel et l'analyse sphérique (Teller, 2003), tout comme les champs d'isovists (Benedikt, 1979) utilisés dans le cadre de ce travail, sont $a$ contrario des approches immergées. Elles permettent d'identifier l'espace ouvert environnant tout point de vue. Pour chaque position ponctuelle, un ensemble de grandeurs morphométriques permettant de quantifier les espaces ouverts est ainsi calculé.

\subsection{Méthode d'analyse des comportements de mobilité}

Comment acquérir de la connaissance sur la prise de décision et, plus particulièrement, sur ce qui motive les choix d'itinéraires dans l'environnement urbain? L'analyse des comportements peut se référer au paradigme des préférences des individus pour une option de choix donnée, sur la base de ses caractéristiques. Deux méthodes sont habituellement utilisées pour étudier les préférences individuelles. Soit on demande aux individus d'interpréter leurs actes, avec les risques d'erreurs que supposent cette introspection et la nécessité de verbaliser pour le sujet étudié, soit on extrapole les préférences en interprétant l'action recensée, ce qui repose sur une hypothèse rationaliste forte.

La technique des préférences déclarées permet d'estimer les préférences des individus à partir d'enquêtes portant sur les déterminants des choix (Hensher, 1994 ; Louvière et al., 2000). Les individus sont directement questionnés sur les motivations de leur choix, soit dans une optique rétrospective (choix déjà effectués), soit dans une optique prédictive (choix futurs). Cette méthode consiste par exemple à travailler sur des parcours commentés pour «caractériser les modes d'appréhension des phénomènes d'ambiance » ainsi que les significations que leur donnent les individus (Péneau, 2000). Aussi intéressante soit elle, cette méthode

6. Sarradin (2004), d'après les travaux de Peponis et al. (1997), rapporte que le champ visuel d'un observateur en mouvement dans l'espace urbain est exposé à deux types de changements. Ainsi, le changement continu ou changement de perspective des surfaces ne provoque aucune réelle modification de la topologie (aussi appelée aspect) de son univers visuel. A contrario, l'apparition ou la disparition d'éléments spatiaux (comme par exemple des extrémités de murs), aussi appelée changement discret, provoque une modification substantielle de la topologie (un événement visuel) de son univers visuel, par introduction d'une discontinuité visible. Partant de ce constat, les auteurs proposent deux méthodes de partitionnement déterministe de l'espace, les s- et e-partitionnements, en fonction des événements visuels et selon une configuration de zones convexes. 
semble peu adaptée aux indices morphométriques étudiés dans le cadre de ce travail. En effet, les individus n'ont pas toujours conscience des attributs qui motivent leurs choix passés ni de leurs rôles respectifs (Costermans, 2001). Ainsi, un décalage est fréquemment observé entre préférences déclarées issues de l'évaluation effectuée par les individus et comportement réels (Gärling et Friman 2002 ; Golledge, 1999). Ces restrictions ont été relevées dans le cadre d'études portant sur des caractéristiques plus classiques reposant sur des attributs concrets des espaces urbains. Elles seraient d'autant plus prégnantes que nous travaillons, dans le cas présent, sur une caractérisation ambiantale beaucoup plus spécifique.

Pour cette raison, l'approche retenue consiste à étudier les choix d'itinéraires, ces choix étant susceptibles de révéler les préférences des piétons. Cette technique des préférences révélées passe par le recueil d'informations indirectes sur les choix effectués, ce qui minimise l'influence que peut avoir l'enquêteur sur le sujet d'étude. Elle repose sur l'hypothèse que les comportements observés, les lieux fréquentés, sont de bons révélateurs des préférences des individus et permettent de mettre en évidence, au moyen d'approches modélisatrices, les caractéristiques significatives discriminant les choix effectués. Cela signifie, selon la théorie économique néoclassique, que les individus sont rationnels et qu'ils agissent conformément à leurs préférences, conscientes ou non. Chaque individu utilise, selon cette approche, une fonction d'utilité qui lui permet d'ordonner les différentes alternatives qui s'offrent à lui dans une situation de choix donnée. Cette méthode économique peut sembler très éloignée de l'approche traditionnelle des ambiances urbaines. Toutefois, l'utilité d'une alternative n'est que la transcription, certes très objective, des préférences qui s'expriment sur les attributs, potentiellement très variés, des alternatives de choix. Au final, l'hypothèse la plus forte est associée au fait que les individus sont censés être capables d'évaluer la satisfaction que peut leur procurer l'usage de telle ou telle option de choix et de sélectionner ensuite celle qui leur paraît la plus satisfaisante.

Dans cette optique, nous proposons de comparer l'espace de mobilité a priori accessible pour chaque individu aux portions d'espaces effectivement empruntées pour identifier les préférences collectives associées aux choix effectués. Nous partons donc de l'hypothèse que l'hétérogénéité ambiantale des rues préside aux choix d'itinéraires des individus. Selon cette hypothèse, les piétons valorisent positivement ou négativement certains espaces et effectuent leurs choix en conséquence. Par l'analyse empirique des itinéraires piétonniers, on peut ainsi identifier les choix effectués et déterminer les ambiances favorables ou non aux déplacements. Il est possible de citer quelques précédents même si les expériences en la matière restent rares. Certaines études ont porté sur l'influence de la végétation sur la fréquence et la portée des déplacements (Shriver, 1997) ou sur les choix d'itinéraires dans des lieux spécifiques comme les jardins publics (Loiteron et Bishop, 2005). Par ailleurs, les travaux de Zacharias (1997, 2006) montrent l'impact de nombreux stimuli visuels sur les choix d'itinéraires des piétons. Mais, à notre connaissance, cette approche comportementale est plus rarement mise en œuvre avec des données d'ambiance morphologiques. 


\section{Présentation du terrain d'étude et des données}

Le quartier de Hautepierre compte environ 14000 habitants. Typique des grands ensembles des années 60-70, il est actuellement en cours de rénovation urbaine ce qui pose avec acuité la question des ambiances à recréer. Situé à environ 4 kilomètres du centre de Strasbourg, à la périphérie ouest, Hautepierre est structuré à partir d'un réseau maillé hexagonal (hexagone de $225 \mathrm{~m}$ de côté - figure 1) déterminant des unités de voisinage de 13 à 14 hectares desservies par des voies unidirectionnelles de desserte. À l'intérieur des mailles, on trouve des cheminements piétons et des espaces verts, la circulation automobile de transit s'effectuant en périphérie. Les mailles rassemblent, pour la plupart, des équipements de proximité (crèches, écoles, centres culturels et associatifs, gymnases...). Socialement, les mailles se caractérisent par une certaine homogénéité, ce qui permet d'isoler plus facilement l'influence de la morphologie.

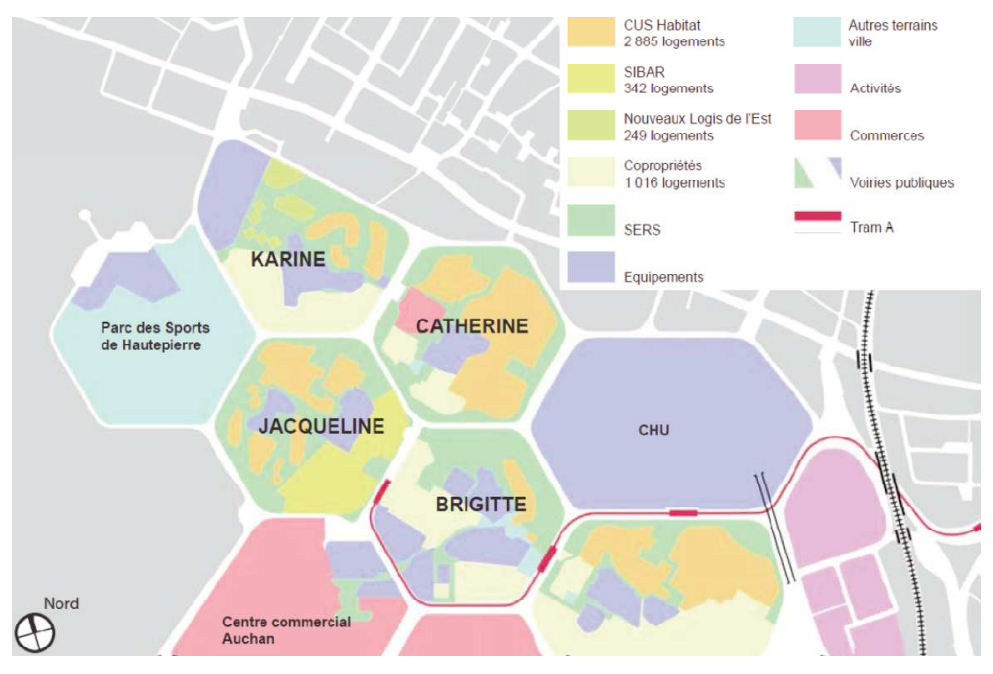

Figure 1. Le quartier de Hautepierre à Strasbourg

Une enquête spécifique a été menée dans ce quartier dans le cadre d'un programme de recherche portant sur les ambiances urbaines ${ }^{7}$. Pour recueillir les comportements de déplacements, nous avons choisi d'enquêter deux cohortes d'individus, des passants (n'habitant pas Hautepierre mais fréquentant régulièrement le quartier pour divers motifs) et des habitants de Hautepierre. Les passants et habitants ont eu à réaliser un trajet dans le quartier de Hautepierre à partir d'un même point de départ, choisi en fonction de sa centralité et du potentiel d'accès aux différentes mailles du quartier. Tous les sujets enquêtés ont dû rejoindre

7. Recherche exploratoire Ambioflux, PIRVE 2008-2010 (Programme interdisciplinaire de recherches Ville et Environnement) cofinancée par le CNRS et le MEDDTL 
une même destination prédéfinie en choisissant l'itinéraire qui leur convenait le mieux (figure 2).

De nombreuses informations ont été recueillies lors de ces trajets (discours, conduites de déplacement, photographies d'éléments d'ambiances prises par les enquêtés) et au cours d'entretiens post-trajets afin de mieux cerner les perceptions des sujets en situation de mobilité (Woloszyn et al., 2011). Ceci explique que les effectifs enquêtés sont relativement restreints : 16 déplacements ont ainsi été relevés ( 8 sont le fait d'habitants du quartier et 8 de passants). Sur la base de ces effectifs nous proposons, comme nous l'avons évoqué, de n'utiliser pour les besoins de cet article que les itinéraires recensés lors de l'enquête. Les indices morphométriques seront intégrés dans un modèle de choix discret afin de mettre en évidence les préférences ambiantales révélées par les trajets choisis.

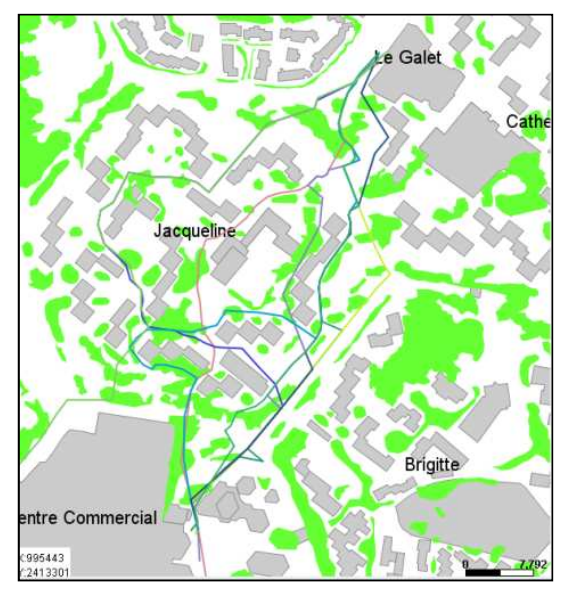

Figure 2. Quelques itinéraires recensés lors de l'enquête

\section{Méthodes}

Comme mentionné précédemment, nous cherchons à produire un ensemble de grandeurs morphométriques décrivant l'environnement proche du piéton pour chacun des itinéraires empruntés ou potentiellement empruntables. A ce niveau d'échelle, on s'intéresse à l'environnement immédiat qui entoure l'individu dans l'espace public. Cet environnement se caractérise par un continuum visuel et peut donc être appréhendé instantanément par les individus.

\subsection{Caractérisation morphologique de l'environnement ambiant du piéton}

Pour calculer un certain nombre d'indicateurs morphologiques censés traduire « objectivement » la configuration des lieux étudiés, les données d'entrée utilisées 
ont été les suivantes. Les empreintes au sol des bâtiments ont été obtenues à partir de la couche bâtiments de la BD Topo ${ }^{\circledR}$ de l'IGN. Les empreintes au sol des espaces verts ainsi que le réseau viaire de la zone d'étude, d'une longueur totale de 22,7 km, sont issus de la BD Topo® et ont été complétés par des données récupérées à partir d'ortho-photographies.

A partir de ces données, un processus en 4 étapes a été mis en œuvre.

- les 402 tronçons du réseau viaire de Hautepierre ont été discrétisés (i.e. convertis en 2469 points distincts) par pas de discrétisation décamétrique (résolution de $10 \mathrm{~m}$ );

- pour chacun des points du réseau discrétisé, deux isovists ont été calculés. Le premier a été calculé à $360^{\circ}$ alors que le second a été restreint à $104^{\circ}$ pour retranscrire la visibilité dans le sens de déplacement des individus. Pour la détermination des bassins de visibilité, nous avons limité la portée du rayon de visibilité à $300 \mathrm{~m}$. Nous estimons en effet empiriquement, sans avoir effectué d'analyse de sensibilité, qu'étant donné le contexte urbain particulier de cette étude (grands ensembles, mailles hexagonales de $225 \mathrm{~m}$ de côté, présence de véhicules, de mobilier et de végétation susceptibles de restreindre le champ de vision d'un adulte), cette distance est un maximum au-delà duquel l'évaluation de la forme de l'espace ouvert immédiatement environnant, par un sujet en situation, n'est pas significativement modifiée.

- chaque isovist a ensuite été « évalué », d'un strict point de vue morphologique, à l'aide de 24 indices de formes auxquels s'ajoutent quatre indices plus spécifiques à connotation paysagère ;

- afin que ces résultats intermédiaires puissent-être réutilisés dans l'étape ultérieure de modélisation des choix, les données morphologiques associées à chacun des 2469 points de discrétisation ont ensuite été agrégées aux tronçons ${ }^{8}$. La figure 3 récapitule le processus mis en œuvre.

8. L'agrégation a été mise en œuvre sur la base d'un calcul de moyenne. D'autres méthodes d'agrégation (écart-type, médiane...) ont été testées. Nous ne présenterons pas ces résultats par souci de concision et parce que les résultats observés diffèrent peu de ceux présentés ciaprès. 


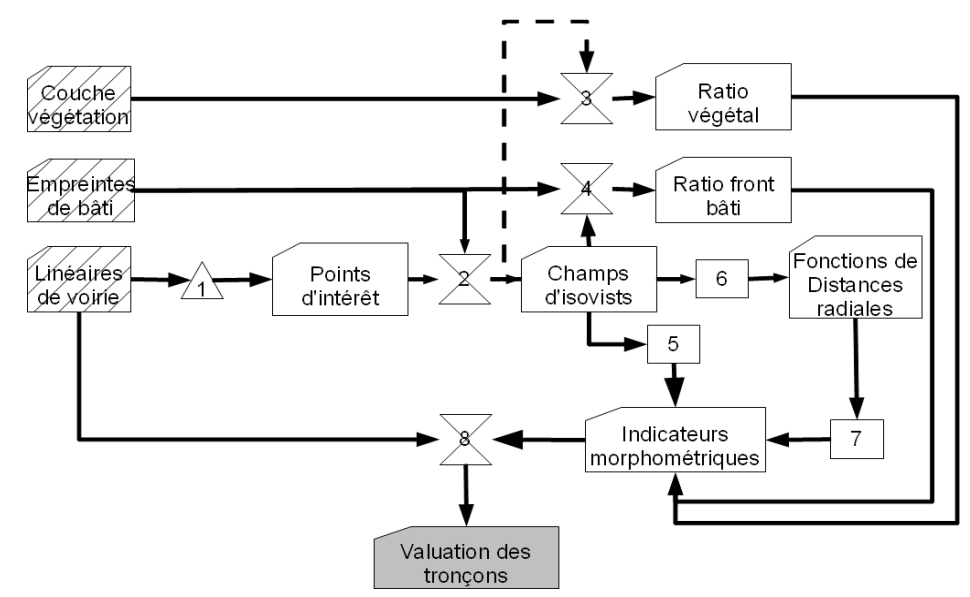

Figure 3. Processus de production des indicateurs morphométriques agrégés au tronçon.

Les descripteurs morphométriques ou indices de formes que nous utilisons sont de plusieurs natures. Certains sont génériques et peuvent être appliqués à n'importe quelle forme polygonale, c'est par exemple le cas du périmètre, de l'aire, du nombre de côtés. Pour caractériser une forme polygonale, l'usage veut qu'elle soit rapportée à différentes formes englobantes ou inscrites (Lee et Sallee, 1970) qui permettent de calculer des indices différenciés du point de vue des ambiances. Sont ainsi utilisés successivement l'enveloppe convexe de la forme (ou plus petit convexe qui contient la forme), le rectangle englobant d'aire minimale (ou rectangle de plus petite aire contenant la forme) ainsi que le plus petit disque englobant. D'autres descripteurs tirent partie de la caractéristique topologique intrinsèque de l'isovist. En effet, ce dernier est, par essence, un polygone étoilé par rapport à un point d'observation. Il présente ainsi une propriété de convexité locale. Plus précisément, le segment reliant ce point de vue à tout point de l'isovist est inclus dans le polygone. Du fait même de cette propriété topologique, nous pouvons caractériser la forme à partir d'une description de son contour par fonction de distances radiales. Cette fonction de distance radiale associe à un azimut donné (issu de la discrétisation par abscisse angulaire) la longueur du rayon reliant le point de vue au point de contour correspondant. L'ensemble des indices présentés sont numérotés pour faciliter la lecture des résultats des modèles de choix discrets qui seront exposés dans la partie 5.

\subsubsection{Mesures de formes simples}

Le périmètre de l'isovist (1). Pour Benedikt (1979), le périmètre de l'isovist, qui correspond à la longueur cumulée des arêtes (bâties ou non) délimitant l'espace vu depuis un point donné, est une des grandeurs permettant d'évaluer la complexité du bassin de visibilité correspondant. Ce périmètre permet notamment d'évaluer la 
quantité de surfaces bâties (longueur du déroulé de façades - indicateur dit d'occlusivité dans cette même référence) vues depuis un point donné. L'aire de l'isovist (2) permet de quantifier la surface d'espace non bâti englobant immédiatement le point de vue (et ainsi directement visible depuis ce même point). Le nombre de côtés de l'isovist (3). Ce nombre, lorsqu'il est élevé, révèle une complexité du contour.

Ces indices de formes simples sont complétés par des indices de compacité qui permettent de comparer la forme de l'isovist à une forme de référence. Les mesures de compacité présentées ci-après sont abondamment discutées dans la littérature. A titre d'illustration, nous nous limiterons à mentionner MacEachren (1985) ou, plus récemment, Maignant (2005).

\subsubsection{Indices de convexité}

Dans le registre des indicateurs génériques de compacité on trouve, pour commencer, les indices de convexité qui permettent de quantifier la déviation de la forme à son enveloppe convexe. La convexité est une notion relativement intuitive (et visuellement perceptible en situation immergée) dans la mesure où elle est une transcription de la présence de renfoncements (ou évidements) et d'encoignures (angles saillants) de bâti dans le champ visuel.

Le nombre de composantes connexes (4) issues de la soustraction de la forme à son enveloppe convexe. Cette grandeur adimensionnée permet de quantifier le nombre de concavités d'un isovist indépendamment de leurs tailles respectives (Thibault et al. 2007). Si ce nombre est nul, la forme est parfaitement convexe. Plus cet indicateur est élevé et plus la forme compte de renfoncements ou encoignures. Ces encoignures sont plus ou moins perceptibles par un observateur en fonction de leurs tailles respectives (figure $4 \mathrm{a}^{9}$ ).

Le degré ou défaut de convexité surfacique (5) correspond au rapport de l'aire de l'objet à l'aire de son enveloppe convexe. Ce rapport a une valeur comprise entre 0 (la forme est globalement filiforme au sens où son épaisseur moyenne est nulle) et 1 (la forme est convexe). A titre d'illustration, le polygone de la figure 4a a un degré de convexité surfacique d'environ 0,38 . Il «occupe » donc concrètement un peu plus du tiers, en surface, de son enveloppe convexe au contour matérialisé par un trait noir. Ce degré correspond à un «taux d'empiétement» des arêtes opaques de bâtiments dans le bassin de visibilité de l'observateur.

Le degré ou défaut de convexité périmétrique (6) est égal au rapport du périmètre de l'enveloppe convexe de l'objet au périmètre de l'objet lui-même. Ce rapport a une valeur comprise entre 0 et 1 (la forme est convexe). Le polygone de la figure $4 \mathrm{a}$ présente un degré de convexité périmétrique d'environ 0,48 . Le périmètre de la forme est donc supérieur au double du périmètre de son enveloppe convexe. Le

9. Cette figure, comme celles qui suivent, a été calculée sur notre terrain d'étude à Hautepierre. 
contour du bassin de visibilité de l'observateur présente donc de nombreuses concavités.

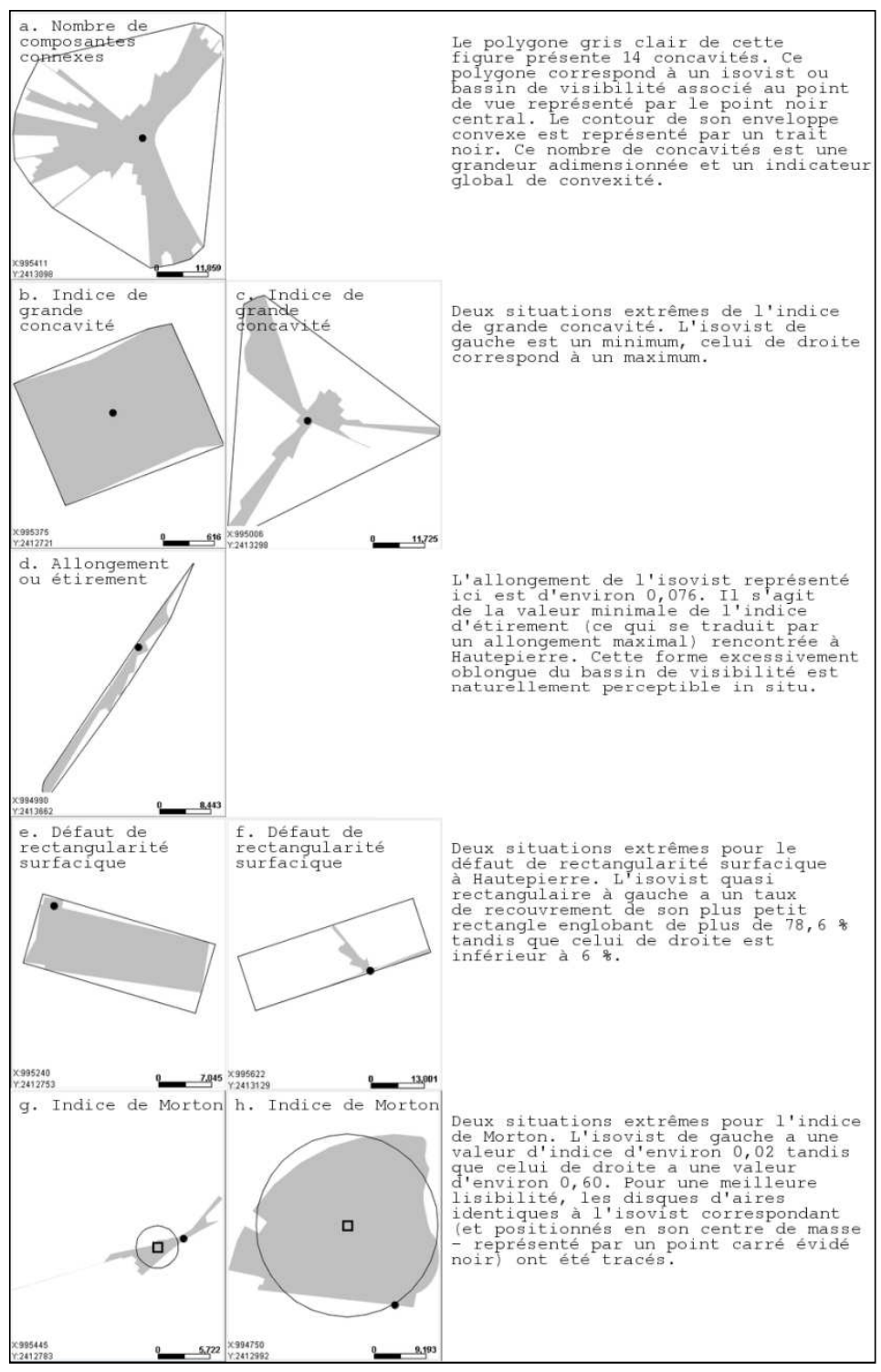

Figure 4. Quelques indices de forme utilisés (1)

L'indice de grande concavité (7) (Bel Hadj Ali, 2001) est une sommation des carrés des aires de chacune des concavités divisée par le nombre de concavités. Sa 
valeur, toujours positive ou nulle (quand la forme est convexe), est d'autant plus importante que la forme présente des concavités de grandes surfaces. Un tel indice de forme permet de compléter utilement les trois indices de convexité déjà présentés dans la mesure où il permet de quantifier les contributions des concavités majeures (figure $4 \mathrm{~b}$ et $4 \mathrm{c}$ ).

L'indice de petite concavité (8) (Bel Hadj Ali, 2001) s'obtient par une sommation de l'inverse des carrés des aires de chacune des concavités divisée par le nombre de concavités. Sa valeur, toujours positive ou nulle (quand la forme est convexe), est d'autant plus importante que la forme présente des concavités de petites surfaces (contour « dentelé »).

\subsubsection{Indices de rectangularité}

Les indices de rectangularité sont des indices de forme qui visent à quantifier la déviation de la forme par rapport à une forme rectangulaire de référence. La rectangularité d'une forme est visuellement perceptible par un observateur in situ dans la mesure où elle s'appuie sur des notions relativement intuitives de parallélisme et de perpendicularité.

L'allongement ou l'étirement (9) (figure 4d) qui correspond au rapport de la longueur du plus petit côté du rectangle englobant d'aire minimale sur la longueur du plus grand côté de ce même rectangle. Cet indicateur varie de 0 (allongement maximal) à 1 (lorsque le rectangle englobant est un carré). Le défaut de rectangularité surfacique (10) correspond au rapport de l'aire de l'isovist à l'aire du rectangle englobant d'aire minimale. Cet indicateur varie de 0 à 1 (lorsque la forme est elle-même rectangulaire - figure $4 \mathrm{e}$ et $4 \mathrm{f})$. Le défaut de rectangularité périmétrique (11) qui correspond au rapport du périmètre de l'objet au périmètre du rectangle englobant d'aire minimale. Ce nouvel indicateur de rectangularité est très différent du précédent. Il n'est pas contraint dans l'intervalle allant de 0 à 1 .

\subsubsection{Indices de circularité}

Les indices de circularité sont des indices qui visent à quantifier la déviation de l'isovist par rapport à une forme circulaire. Ces indices peuvent être rapprochés de l'inégalité isopérimétrique. Dans le plan, celle-ci établit que, pour une valeur de périmètre donnée, la surface associée qui maximise la valeur de l'aire est un cercle. La circularité d'une forme est une notion relativement intuitive dans la mesure où elle est une traduction de la notion d'équidistance de l'ensemble des points et arêtes d'un contour à un point central donné. Elle est par contre moins visuellement perceptible en situation immergée que l'indicateur de dérive qui sera présenté ultérieurement car le point d'observation ne coïncide pas nécessairement avec le centre de masse. 
L'indice de Miller (12) (Miller, 1953) ou déficit isopérimétrique. Cet indice de compacité, calculé à partir de la formule de l'équation (1), a une valeur comprise entre 0 (circularité minimale, le périmètre est très grand et l'aire englobée très petite) et 1 (circularité maximale, lorsque l'isovist est un disque). Il est, comme les indices de Morton et Gravélius, sans dimension.

$$
I_{\text {Miller }}=\frac{4 \pi \times \text { aire }}{\text { périmètre }^{2}}
$$

L'indice de Morton (13) ou indice de circularité (figure $4 \mathrm{~g}$ et $4 \mathrm{~h}$ ) correspond au rapport de l'aire de l'objet à l'aire d'un disque qui aurait le plus grand axe de l'isovist pour diamètre (cf. équation (2). Comme l'indice de Miller, cet indice varie de 0 à 1 (forme circulaire).

$$
I_{\text {Morton }}=\frac{4 \times \text { aire }}{\pi \times L_{a p}^{2}}
$$

L'indice de Gravélius (14) est un indicateur de circularité qui correspond au rapport formulé dans l'équation (3). Comme constaté par Maignant (2005), cet indice varie de 1 (forme circulaire) à l'infini, dans le sens inverse des indices de Miller et Morton.

$$
I_{\text {Gravélius }}=\frac{\text { périmètre }}{\sqrt{4 \times \pi \times \text { aire }}}
$$

Le plus grand diamètre inscrit centré au point d'observation (15). Cet indice de forme, étroitement corrélé à la position du point de vue, permet d'évaluer l'éloignement du point d'observation à l'arête opaque la plus proche (figure 5a et 5b). Il est visuellement perceptible par un observateur in situ et repose sur la perception de la distance aux façades bâties.

Le jaggedness (16). Cet indicateur de circularité mis en œuvre dans (Franz et Wiener, 2005) correspond, à un facteur multiplicatif près, à l'inverse de l'indice de Miller. Cette grandeur adimensionnée, comme présentée dans l'équation (4), n'est pas normalisée.

$$
\text { Jaggedness }=\frac{\text { périmètre }^{2}}{\text { aire }}
$$

Comme constaté dans Thibault et al. (2007), tous ces indices sont particulièrement efficaces pour des formes faiblement non-convexes. Ils le sont nettement moins pour des formes fortement non-convexes comme les isovists qui correspondent parfois à des polygones très étoilés. 


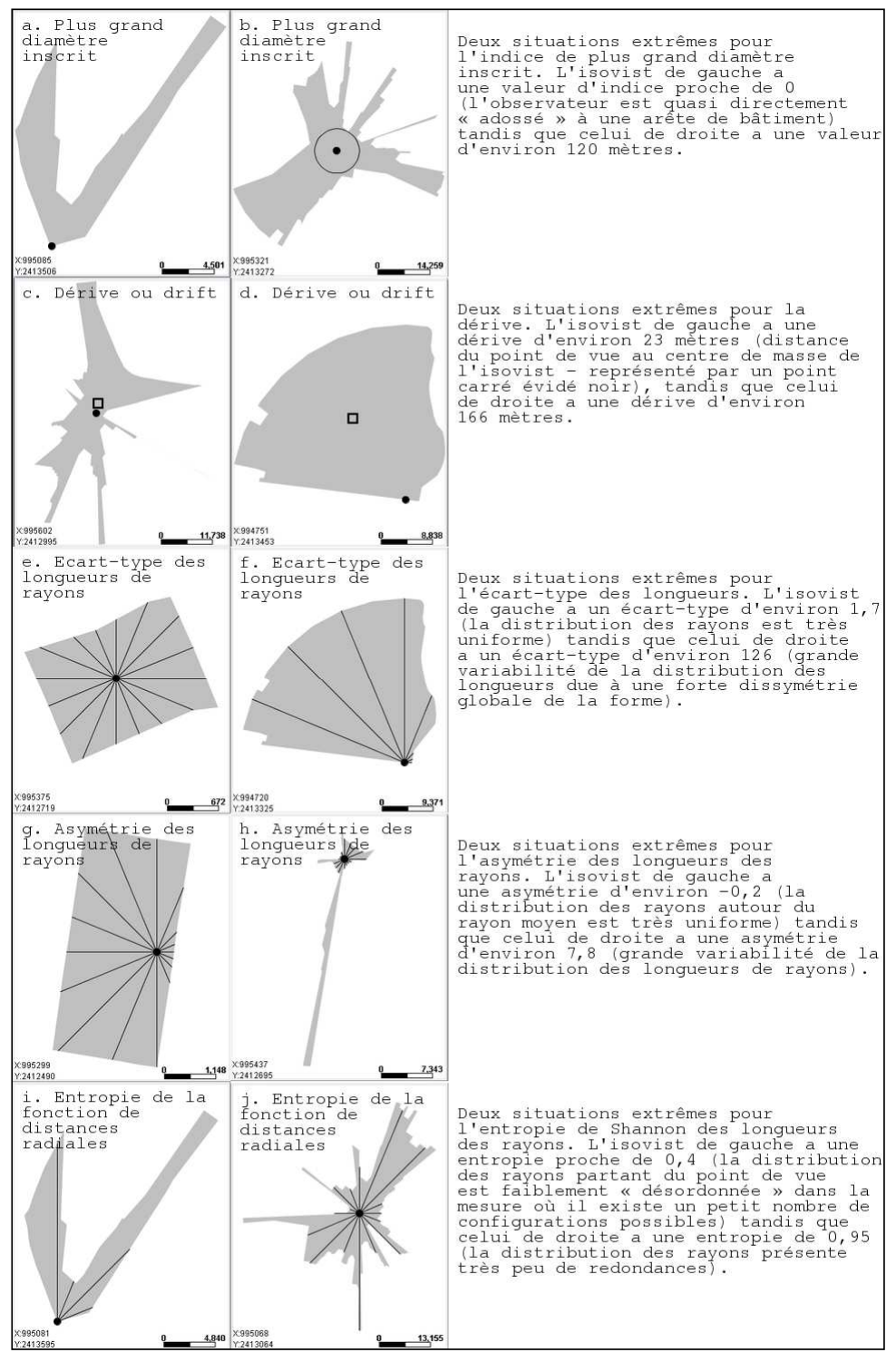

Figure 5. Quelques indices de forme utilisés (2) 


\subsubsection{Indices spécifiques aux formes étoilées ${ }^{10}$}

Un polygone est étoilé en un point donné (ce point est, dans le cas précis d'un isovist, appelé point de vue ou point de génération) lorsque tout segment reliant ce point à n'importe quel autre point de la forme est contenu dans le polygone. Du fait de cette propriété topologique, une telle forme peut-être décrite par la seule transcription de son contour en une fonction de distances radiales. Cette fonction de distance radiale associe à un azimut donné (issu de la discrétisation par abscisse angulaire) la longueur du rayon reliant le point de vue au point de contour correspondant. La majeure partie des indices de forme présentée ci-après analyse cette fonction de distances radiales comme une simple distribution d'une variable réelle.

La dérive (17) (Conroy Dalton et Dalton, 2001) correspond à la distance (en mètres) du point de vue au centre de masse de l'isovist correspondant. Cette grandeur permet de mettre en perspective un point de vue d'une part et un centre de masse qui minimise la longueur de rayons, d'autre part, c'est-à-dire de quantifier l'impression éventuelle de l'observateur d'être, ou non, situé au centre de son bassin de visibilité (figure $5 \mathrm{c}$ et $5 \mathrm{~d}$ ).

Le rayon moyen (18) (Benedikt, 1979) correspond à la moyenne arithmétique des longueurs des rayons reliant le point de vue à tout point du contour. Cet indice de forme est une indication de la dimension globale de la forme. Pour le calculer, nous avons arbitrairement discrétisé le polygone en 512 rayons ou abscisses angulaires équi-répartis. Le rayon minimum (19) équivaut à la plus petite longueur des rayons reliant le point de vue à tout point du contour. Le rayon maximum (20) équivaut à la plus grande longueur des rayons reliant le point de vue à tout point du contour. L'écart-type des longueurs de rayon (21) (Benedikt, 1979) permet de caractériser la dispersion de ces longueurs par rapport à la longueur moyenne (figure $5 \mathrm{e}$ et $5 \mathrm{f}$ ). Il s'agit ici d'un indicateur d'étalement. Plus cet écart-type est élevé et plus la distribution des rayons est étalée. L'asymétrie des longueurs de rayon (22) (Benedikt, 1979) est un estimateur, issu de la statistique, de dissymétrie de la densité de probabilité d'une distribution de longueurs radiales. Elle permet, avec un point de vue très anthropocentrique (dans le sens où cette grandeur est relative à la position de l'observateur), d'identifier les formes qui présentent une évidente irrégularité dans la distribution des rayons d'échantillonnage, c'est-à-dire une irrégularité dans l'éloignement du contour au point de vue (figure $5 \mathrm{~g}$ et $5 \mathrm{~h}$ ).

Le kurtosis des longueurs de rayon (23) est un estimateur, issu de la statistique, d'aplatissement de la densité de probabilité d'une distribution de longueurs radiales. Cet indicateur permet principalement d'identifier la présence, dans la distribution, de grands écarts à la valeur moyenne (il s'agit en effet d'un moment de variable centrée réduite élevé à la puissance $4^{\text {ème }}$ ). D'un point de vue anthropocentrique, et même s'il ne s'agit pas d'un indicateur d'asymétrie, un point de vue excentré (tel celui où l'observateur est bordé d'un mur d'un côté et d'une vue dégagée de l'autre) produit une valeur de Kurtosis élevée. Cette valeur de Kurtosis est aussi élevée si

10. Ces indices n'ont été calculés qu'à partir des isovists à $360^{\circ}$. 
l'observateur est placé au centre d'un rond point, lieu de convergence de plusieurs allées rayonnantes. A contrario, une position centrale sur une place circulaire sans dégagement correspond à un faible Kurtosis. Cette grandeur permet donc d'identifier les formes pour lesquelles la distribution des longueurs de rayon est plutôt pointue en la moyenne. Elle a été mise en œuvre dans Leduc et al. (2011).

L'entropie de Shannon de la fonction des distances radiales (24) est un indice à caractère informationnel ${ }^{11}$ (Stamps III, 2002, 2005, 2010 ; Woloszyn et Leduc, 2011). Cette grandeur correspond à une évaluation de la prévisibilité du contour, c'est-à-dire à une évaluation du nombre de configurations possibles pour les longueurs de rayons (figure $5 \mathrm{i}$ et $5 \mathrm{j}$ ). A titre d'exemple, un disque vu depuis son centre présente une entropie nulle dans la mesure où il n'y a aucune incertitude sur la longueur des rayons reliant le point de vue au contour. A contrario, dans un environnement urbain complexe aux arêtes bâties multiples provoquant de nombreux effets de masque peu ordonnés, le contour de l'isovist est «imprévisible », complexe, et l'entropie de la fonction des distances radiales tend vers 1.

\subsubsection{Indices à connotation paysagère}

Pour compléter ces indices morphométriques, nous avons inclus quelques indices à connotations paysagère, toujours sur la base des champs de visibilité.

La quantité de surface végétale (25) contenue dans l'isovist. Le ratio de surface végétale (26) contenue dans l'isovist rapportée à la surface totale. Dans le cas précis du terrain d'étude de Hautepierre, cette valeur de ratio est comprise entre 0 et $57 \%$. La longueur de front bâti visible (27) depuis un point d'observation. Le ratio de longueur de front bâti (28) rapportée au périmètre total de lisovist. Cet indicateur d'« occlusivité » est directement perceptible par un piéton in situ. Il permet de distinguer, pour le contour immédiatement perçu, la part de façades «opaques » des autres effets de masque.

Bien entendu ces indicateurs, zénithaux par leur construction, ne constituent qu'une approximation de ce que les piétons peuvent percevoir. D'une part, ils sont restrictifs par rapport au contenu ambiantal que les individus appréhendent et perçoivent, d'autre part ils sont beaucoup plus précis que ne le sont les sens humains. Ils permettent cependant de retranscrire en partie l'environnement morphologique immersif immédiatement perceptible par les individus. L'ensemble de ces indices morphométriques (tableau 1) caractérisant les espaces accessibles pour les piétons va donc être intégré au sein de modèles de choix discret ce qui permettra d'identifier les caractéristiques morphologiques favorables aux pratiques de déplacements pédestres. Notre parti pris repose sur l'analyse de tous ces indices, sans a priori malgré les similitudes existantes entre certains de ces indices, le modèle de choix étant capable de discerner les éventuelles redondances d'indices pour ne sélectionner que ceux qui sont significatifs.

11. Cet indice n'est calculé qu'à partir des isovists à $360^{\circ}$. 
Tableau 1. Récapitulatif des 7 grandes familles d'indicateurs mises en æuvre

\begin{tabular}{|c|c|c|}
\hline Catégorie d'indicateurs & & Liste d'indicateurs associés \\
\hline $\begin{array}{l}\text { Indices simples de } \\
\text { formes polygonales }\end{array}$ & $\begin{array}{l}1 \\
2 \\
3\end{array}$ & $\begin{array}{l}\text { Périmètre de l'isovist } \\
\text { Aire de l'isovist } \\
\text { Nombre de côtés de l'isovist }\end{array}$ \\
\hline Indices de convexité & $\begin{array}{l}4 \\
5 \\
6 \\
7 \\
8\end{array}$ & $\begin{array}{l}\text { Nombre de composantes connexes } \\
\text { Défaut de convexité surfacique } \\
\text { Défaut de convexité périmétrique } \\
\text { Indice de grande concavité } \\
\text { Indice de petite concavité }\end{array}$ \\
\hline Indices de rectangularité & $\begin{array}{l}9 \\
10 \\
11\end{array}$ & $\begin{array}{l}\text { Allongement } \\
\text { Défaut de rectangularité surfacique } \\
\text { Défaut de rectangularité périmétrique }\end{array}$ \\
\hline Indices de circularité & $\begin{array}{l}12 \\
13 \\
14 \\
15\end{array}$ & $\begin{array}{l}\text { Indice de Miller } \\
\text { Indice de Morton } \\
\text { Indice de Gravélius } \\
\text { Plus grand diamètre inscrit centré au } \\
\text { point d'observation } \\
\text { Jaggedness }\end{array}$ \\
\hline $\begin{array}{l}\text { Indices spécifiques aux } \\
\text { formes étoilées }\end{array}$ & $\begin{array}{l}17 \\
18 \\
19 \\
20 \\
21 \\
22 \\
23 \\
24\end{array}$ & $\begin{array}{l}\text { Dérive (ou drift) } \\
\text { Rayon moyen } \\
\text { Rayon minimum } \\
\text { Rayon maximum } \\
\text { Ecart-type des longueurs de rayon } \\
\text { Asymétrie des longueurs de rayon } \\
\text { Kurtosis des longueurs de rayon } \\
\text { Entropie de Shannon de la fonction de } \\
\text { distances radiales }\end{array}$ \\
\hline $\begin{array}{l}\text { Indices à connotation } \\
\text { paysagère }\end{array}$ & $\begin{array}{l}25 \\
26 \\
27 \\
28\end{array}$ & $\begin{array}{l}\text { Quantité de surface végétale inscrite } \\
\text { Ratio de surface végétale inscrite } \\
\text { Longueur de front bâti visible } \\
\text { Ratio de longueur de front bâti visible }\end{array}$ \\
\hline
\end{tabular}

\subsection{Principes des modèles de choix discrets}

Le processus d'évaluation qui conduit aux choix d'itinéraires sera étudié au moyen d'un modèle de choix discret et plus spécifiquement du logit multinomial (McFadden, 1981). Ce type de modèle permet d'analyser le choix effectué par chaque individu parmi un ensemble d'alternatives potentielles discriminées sur la base de leurs caractéristiques. Comme le rappelle Bierlaire (1998), les modèles de choix discrets décomposent le processus de choix de la manière suivante.

- Définition des options de choix disponibles pour chaque individu. Les modèles de choix discrets intègrent généralement cette étape qui consiste à éliminer les alternatives non pertinentes en amont de la mise en œuvre du modèle à proprement parlé (Prato, 2009). Cette étape permet de définir un ensemble de choix réduit (de Palma et al., 1994), réaliste, pour décrire le choix tel qu'il émerge dans une situation donnée, tel qu'il est effectué par chaque individu (Besse, 2010 ; Thibaud, 2002). 
Cela permet ensuite d'optimiser le fonctionnement du modèle pour identifier les préférences individuelles de manière fine. Cette démarche doit être rapprochée de l'idée des psychologues selon laquelle il existe un nombre maximal d'alternatives que les individus sont capables d'évaluer de manière simultanée, a fortiori lorsque ces alternatives sont appréhendées sur la base de caractéristiques très spécifiques. Il y a donc une différence entre le nombre total d'itinéraires disponibles (ensemble de choix universel) et le nombre d'alternatives réellement évaluées par chaque individu. La restriction du contexte de choix (Ettema et al., 2011) peut être pilotée par le modélisateur en fonction des hypothèses associées aux comportements de mobilité. Dans tous les cas, l'ensemble de choix fini doit être réaliste c'est-à-dire raisonnable en termes d'alternatives retenues. Dans le cas présent, nous nous restreindrons à des itinéraires potentiels dont la longueur n'excède pas $120 \%$ de celle du trajet choisi par chaque enquêté lors de l'enquête ${ }^{12}$. Ce seuil a été choisi de manière empirique, sur la base des trajets récoltés lors de l'enquête ;

- Caractérisation des itinéraires par des attributs. L'analyse des choix effectués repose sur l'idée que le piéton ne se déplace pas de manière aléatoire dans la ville. Cette rationalité s'exprime de manière large : à une minimisation du temps ou des distances parcourues, le piéton va, en distinguant les «qualités » et «défauts » des lieux potentiellement accessibles, chercher à maximiser la fréquentation des espaces attractifs aux dépens de ceux qui le sont moins. Nous partons donc du principe que tous les itinéraires retenus dans l'ensemble de choix ont initialement la même probabilité d'être empruntés. Nous postulons ensuite, il s'agit là d'une hypothèse forte, que la morphologie bâtie du contexte immédiat de mobilité conditionne le choix d'itinéraire. A chaque instant, le piéton en mouvement perçoit les caractéristiques de son environnement proche, enregistre des séquences de paysage, que les points de mesures morphométriques traduisent en partie (figure 6), et auxquels le piéton va réagir par ses choix d'itinéraires. Les données morphologiques sont agrégées au tronçon ${ }^{13}$ pour permettre ensuite une valuation de chaque itinéraire potentiel. Les itinéraires empruntés sont alors comparés à l'ensemble de trajets potentiels pour mettre en évidence les différences morphologiques sur lesquelles sont censés reposer les choix des piétons en mouvement ;

12. Les itinéraires recensés lors de l'enquête étant très variables en termes de longueur, il y a donc autant d'ensembles de choix que d'enquêtés

13. C'est-à-dire entre deux points de choix d'itinéraire potentiels adjacents 


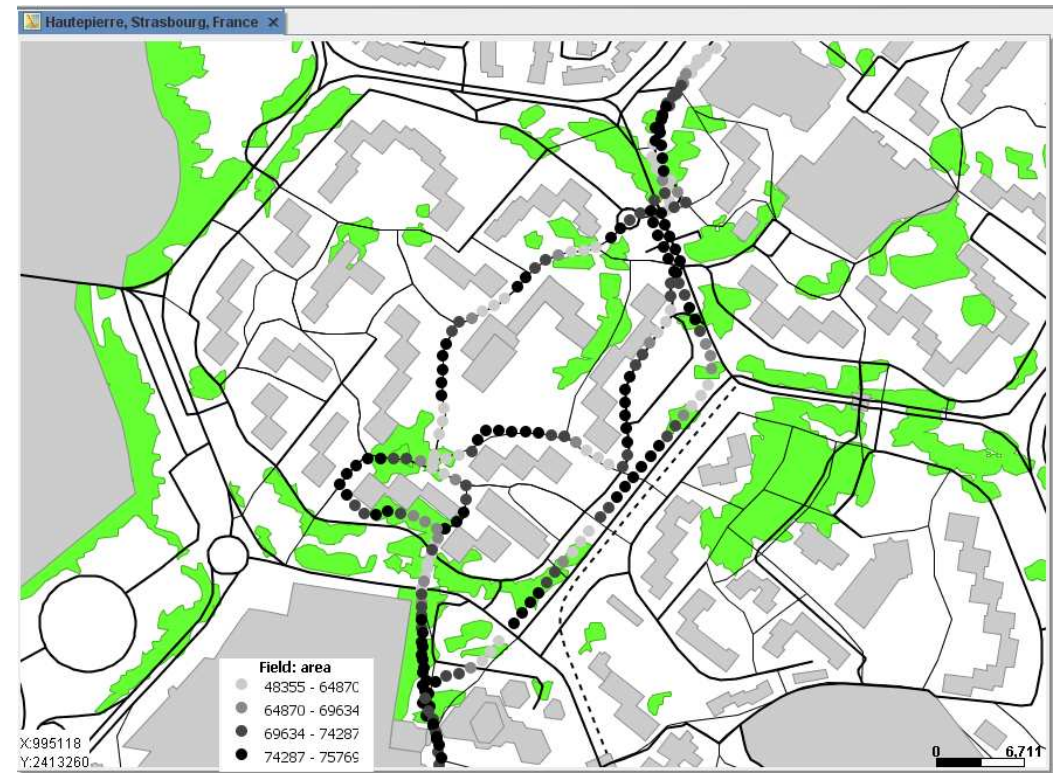

Figure 6. Exemple de discrétisation de trois itinéraires reliant le Centre socioculturel Le Galet au centre commercial dans le quartier de Hautepierre. La symbologie correspond à une classification par intervalle des aires de l'isovist calculée en chaque point considéré.

- Processus et règles de décision de l'individu. Comme nous l'avons mentionné, chaque individu dispose d'une fonction d'utilité qui lui permet de comparer et d'ordonner les différentes alternatives qui s'offrent à lui selon une approche probabiliste (de Palma et Thisse, 1989). Le modèle logit est donc utilisé dans le but de reproduire et d'expliquer le choix d'itinéraire effectué par chaque piéton par le calcul désagrégé de probabilités de choix. Parce que les habitants et passants connaissent et fréquentent régulièrement le terrain d'étude, nous partons du principe que le choix d'itinéraire se fait au départ du trajet et non de proche en proche. Le trajet est considéré comme un tout et les comportements sont analysés de manière globale. La capacité du modèle à reproduire le choix relevé est classiquement mesurée par la statistique du maximum de vraisemblance et par le calcul d'un pseudo- $\mathrm{R}^{2}$ ajusté, ce qui permet de mettre en évidence les préférences des individus pour certaines caractéristiques. Les résultats obtenus se présentent sous la forme d'un vecteur d'attributs auxquels sont attachés des coefficients qui indiquent le sens de la relation et son intensité. Du fait de la forme additive de l'utilité, le choix parmi les options potentielles est compensatoire; un compromis (trade-off) est réalisé entre les attributs pour maximiser l'utilité des individus c'est-à-dire pour choisir l'option qui leur procure une satisfaction maximale. Ce compromis correspond à la meilleure combinaison d'attributs explicatifs mais il peut masquer certaines relations statistiquement significatives. Aussi, il semble utile, dans un premier temps, de 
rechercher les relations univariées entre le choix d'itinéraire et chaque variable morphométrique, même si cette approche est réductrice dans la mesure où elle ne tient pas compte des corrélations entre les variables.

A l'issue de ce processus, les attributs morphométriques ayant joué un rôle dans la prise de décision sont identifiés soit sous la forme de relations univariées (mesure du pouvoir explicatif de chaque variable) soit par le biais d'un modèle multivarié (recherche de la meilleure combinaison de variables). Ces paramètres comportementaux permettent de définir les préférences individuelles pour certaines caractéristiques d'ambiance.

\section{Résultats}

Le modèle logit multinomial a été appliqué à l'ensemble de la population enquêtée puis séparément aux catégories habitants et passants. Cela ne figure pas dans les tableaux suivants mais les premiers résultats montrent que les passants sont moins rationnels dans leurs déplacements que les habitants dans la mesure où la longueur des itinéraires choisis s'écarte significativement du plus court chemin. En effet, les habitants semblent plus «pragmatiques » sans pour autant opter pour l'itinéraire le plus efficient. Ce résultat s'explique peut-être par la pratique régulière des lieux chez les habitants et donc par une connaissance plus poussée des itinéraires de cheminement. Il faut également noter que cette différence est examinée sous l'angle d'une rationalité reposant sur le temps et la distance de déplacement. Or, les préférences ambiantales se réfèrent plutôt à une rationalité cognitive. Ainsi, l'allongement des distances parcourues peut correspondre à la recherche d'ambiances plus favorables par les passants qui sont, par nature, moins habitués au territoire fréquenté et donc plus attentifs aux ambiances rencontrées. Inversement, mais cela reste à confirmer par les modèles d'ambiances, les habitants peuvent renoncer à des ambiances favorables au profit de déplacements plus efficaces du strict point de vue de l'effort fourni ou du temps de parcours.

Examinons les résultats ${ }^{14}$ des modèles de choix univariés pour l'ensemble des personnes enquêtées puis, plus spécifiquement, pour les catégories habitants et passants (tableau 2). Seuls les résultats pour les isovists partiels limités à $104^{\circ}$ (dans le sens du mouvement) sont présentés, hormis pour les indices de formes étoilées uniquement disponibles pour les isovists à $360^{\circ}$. Il existe très peu de différences concernant la significativité des indices et le sens des relations pour ces deux types d'isovists. Cela semble indiquer que le piéton en mouvement a une perception morphologique des espaces qui n'est pas dépendante de son sens de parcours. Les formes bâties sont appréhendées de manière globale sans directionnalité. On notera également qu'il y a des similitudes, en termes de préférences ambiantales, entre habitants et passants alors que, comme nous l'avons évoqué, les itinéraires choisis sont assez différents. Ceci est assez remarquable et pose la question de cette distinction fréquemment évoquée dans les comportements de mobilité. Globalement, les piétons privilégient des espaces ouverts de faible étendue (aire de l'isovist,

14. Les valeurs consignées dans le tableau 2 correspondent au pseudo- $\mathrm{R}^{2}$ ajusté 
longueur des rayons). Les résultats sont en revanche plus discutés en ce qui concerne la complexité des formes. D'un côté, certains indices semblent indiquer que les formes simples, plutôt homogènes, voire circulaires, sont recherchées comme l'indique le sens de la relation entre itinéraires choisis et les indices d'entropie, l'écart-type des longueurs des rayons, les indices de Morton, Miller et Gravélius. D'un autre côté certains indicateurs mettent en évidence une fréquentation de formes plus spécifiques qui engendrent une complexité relative du bassin de visibilité (asymétrie des longueurs de rayons, défaut de convexité surfacique, défaut de rectangularité, kurtosis). Cette contradiction apparente met en évidence une attraction potentielle pour des formes bâties très diversifiées ce qui est extrêmement intéressant. En effet, rares sont les indices morphométriques qui ne sont pas significatifs dans cette approche univariée. Cela signifie qu'il n'existe pas une forme bâtie qu'il faudrait privilégier et que c'est la combinaison de formes bien spécifiques, comme nous le verrons avec les modèles multivariés, qui exercera une attraction sur les individus en mouvement.

De la même manière, les résultats associés aux indices paysagers peuvent sembler surprenants. Certes, la minimisation du bâti peut paraître assez classique mais elle n'est pas compensée par la présence d'espaces verts. Ces résultats doivent toutefois être appréhendés avec précaution puisque l'approche univariée ne permet pas de travailler sur des combinaisons d'indicateurs. On verra d'ailleurs dans les résultats des modèles multivariés que les espaces verts sont recherchés dans des contextes morphologiques bien spécifiques.

Tableau 2. Résultats univariés

\begin{tabular}{|c|c|c|c|c|c|}
\hline & & Indicateurs & $\begin{array}{l}\text { Ensemble } \\
\text { des } \\
\text { enquêtés }\end{array}$ & Habitants & Passants \\
\hline \multirow{3}{*}{$\begin{array}{l}\text { Mesures de } \\
\text { formes } \\
\left(104^{\circ}\right)\end{array}$} & 1 & Périmètre isovist & $-0,1$ & $-0,13$ & $-0,07$ \\
\hline & 2 & Aire isovist & $-0,57 * * *$ & $-0,49 * * *$ & $-0,65 * * *$ \\
\hline & 3 & Nbre côté isovist & $-0,29 * * *$ & $-0,23 * * *$ & $-0,42 * * *$ \\
\hline \multirow{5}{*}{$\begin{array}{l}\text { Indices de } \\
\text { convexité } \\
\left(104^{\circ}\right)\end{array}$} & 4 & Nbre de composantes connexes & $-0,06$ & $-0,09$ & $-0,04$ \\
\hline & 5 & Défaut de convexité surfacique & $-0,96 * * *$ & $-0,92 * * *$ & $-1,01 * * *$ \\
\hline & 6 & Défaut de convexité périmétrique & $-0,24 * * *$ & $-0,19$ & $-0,32 * *$ \\
\hline & 7 & Indice de grande concavité & 0,26 & 0,25 & 0,27 \\
\hline & 8 & Indice de petite concavité & $-4,1$ & $-6,1$ & $-6,1$ \\
\hline \multirow{3}{*}{$\begin{array}{l}\text { Indices de } \\
\text { rectangularité } \\
\left(104^{\circ}\right)\end{array}$} & 9 & Allongement & $-0,1$ & $-0,03$ & $-0,2$ \\
\hline & 10 & Défaut de rectangularité surfacique & $-0,59 * * *$ & $-0,57 * * *$ & $-0,61 * * *$ \\
\hline & 11 & Défaut de rectangularité périmétrique & $-0,07$ & $-0,06$ & $-0,09$ \\
\hline \multirow{3}{*}{$\begin{array}{l}\text { Indices de } \\
\text { circularité } \\
\left(104^{\circ}\right)\end{array}$} & 12 & Indice de Miller & $-1,78 * * *$ & $-3,71 * * *$ & $-1,26 * * *$ \\
\hline & 13 & Indice de Morton & $-0,34 * * *$ & $-0,27 * * *$ & $-0,44 * * *$ \\
\hline & 14 & Indice de Gravélius & $1,19 * * *$ & $1,38 * * *$ & $1,05 * * *$ \\
\hline
\end{tabular}




\begin{tabular}{|c|c|c|c|c|c|}
\hline & $\begin{array}{l}15 \\
16\end{array}$ & $\begin{array}{l}\text { Plus grand diamètre inscrit } \\
\text { Jaggedness }\end{array}$ & $\begin{array}{l}-0,42 \\
1,09 * * *\end{array}$ & $\begin{array}{l}-0,78 \\
1,31 * * *\end{array}$ & $\begin{array}{l}-0,13 \\
0,96 * * *\end{array}$ \\
\hline \multirow{8}{*}{$\begin{array}{l}\text { Indices de } \\
\text { formes } \\
\text { étoilées } \\
\left(360^{\circ}\right)\end{array}$} & & Dérive & $0,60 * * *$ & $0,56^{* * *}$ & $0,63 * * *$ \\
\hline & & Rayon moyen & $-0,56 * * *$ & $-0,49 * * *$ & $-0,66^{* * *}$ \\
\hline & 19 & Rayon minimum & $-0,29 * * *$ & $-0,23$ & $-0,35^{* *}$ \\
\hline & 20 & Rayon maximum & $-3,54 * * *$ & $-3,49 * * *$ & $-3,65$ \\
\hline & 21 & Ecart-type des longueurs de rayon & $-3,56 * * *$ & $-4,31 * * *$ & $-3,01 * * *$ \\
\hline & 22 & Asymétrie des longueurs de rayon & $1,01 * * *$ & $0,85 * * *$ & $1,45^{* * *}$ \\
\hline & 23 & Kurtosis des longueurs de rayon & $1,42 * * *$ & $1,16^{* * *}$ & $2,00 * * *$ \\
\hline & 24 & Entropie des distances radiales & $-0,79 * * *$ & $-0,69 * *$ & $-0,91 * * *$ \\
\hline \multirow{4}{*}{$\begin{array}{l}\text { Indices } \\
\text { paysagers } \\
\left(104^{\circ}\right)\end{array}$} & 25 & Qté de surface végétale & $-0,36 * * *$ & $-0,35 * * *$ & $-0,38 * * *$ \\
\hline & 26 & Ratio de surface végétale & $-0,17$ & 0,03 & $-0,43$ \\
\hline & 27 & Longueur de front bâti & $-0,33 * * *$ & $-0,27 * * *$ & $-0,44 * * *$ \\
\hline & 28 & Ratio de longueur de front bâti & $-1,1 * * *$ & $-0,96 * *$ & $-1,22 * * *$ \\
\hline
\end{tabular}

Les résultats du tableau 3 présentent la meilleure combinaison possible d'indices morphologiques pour chaque catégorie de piétons. Chacun de ces modèles multivariés synthétiques retient, au maximum, trois indices explicatifs. Au-delà, les autres indices n'apportent pas de plus-value pour expliquer les choix de cheminement des enquêtés. Ce nombre restreint d'indices significatifs mis en évidence par les modèles peut s'expliquer par le faible nombre d'enquêtés, ce qui laisse peu de latitude pour l'amélioration de la statistique du maximum de vraisemblance. Mais ces modèles très synthétiques sont également assez logiques puisque l'on imagine difficilement que les choix d'itinéraires soient guidés par une combinaison très complexe d'indices morphologiques.

Les résultats pour l'ensemble des enquêtés (habitants et passants) montrent une relation positive au ratio de surface de végétation et négative à l'aire de l'isovist. Les trajets semblent se construire selon une succession d'espaces ouverts de faible étendue tout en optimisant le contact visuel avec les espaces verts. Ce rôle positif de la végétation démontre que les espaces verts constituent un élément important d'aménagement urbain et de valorisation des lieux mais pas dans n'importe quel type d'espace, morphologiquement parlant. En effet, alors que la végétation n'apparaît pas comme un élément significatif dans l'approche univariée, on peut remarquer qu'elle devient prépondérante dans des espaces qui sont caractérisés par une association d'indices spécifique. C'est là tout l'intérêt des modèles multivariés qui permettent de définir des types d'espaces attractifs. Seul, le végétal ne présente pas un intérêt particulier pour les piétons alors qu'il en aura dans des configurations bâties particulières. On remarquera d'ailleurs que, si la végétation a un impact positif, celle-ci ne s'inscrit pas dans les mêmes types d'espaces selon la catégorie d'enquêtés que l'on étudie. Pour l'ensemble des enquêtés, il s'agit d'espaces ouverts de faible étendue et relativement homogènes, circulaires alors que pour les 
habitants, l'impact positif de la végétation s'inscrit dans des espaces peu étendus et aux contours plus spécifiques (circularité minimale et grand périmètre de l'isovist).

Tableau 3. Résultats des modèles multivariés

Ensemble des enquêtés

\begin{tabular}{|c|c|c|c|}
\hline Indicateurs & Angle Isovist & Coeff. & $\begin{array}{c}\text { Log- } \\
\text { Vraisemblance }\end{array}$ \\
\hline 21. Ecart-type des longueurs de rayon & $360^{\circ}$ & $-1,81 * * *$ & $-31,25$ \\
\hline 26. Ratio de surface végétale & $360^{\circ}$ & $4,49 * * *$ & $-25,92$ \\
\hline 2. Aire isovist & $104^{\circ}$ & $-0,71 * *$ & $-23,57$ \\
\hline Log-Vrais. initiale $\quad-54,74$ & $* * p<0,05$ & $* * * p<0,01$ & \\
\hline $\begin{array}{ll}\text { Log-Vrais. finale } & -23,57 \\
\text { Pseudo- } R^{2} \text { ajusté } & 0,51\end{array}$ & & & \\
\hline
\end{tabular}

\section{Habitants}

\begin{tabular}{llll}
\hline Indicateurs & Angle Isovist & Coeff. & $\begin{array}{l}\text { Log- } \\
\text { Vraisemblance }\end{array}$ \\
\hline 14. Indice de Gravélius & $104^{\circ}$ & $1,99 * * *$ & $-16,13$ \\
26. Ratio de surface végétale & $104^{\circ}$ & $2,42 * * *$ & $-12,37$ \\
2. Aire isovist & $104^{\circ}$ & $-0,52$ & $-11,82$ \\
\hline Log-Vrais. initiale -29,19 & $* * p<0,05$ & $* * * p<0,01$ & \\
Log-Vrais. finale -11,82 & & & \\
Pseudo- $R^{2}$ ajusté 0,51 & & &
\end{tabular}

\begin{tabular}{llll} 
Passants & & \\
\hline Indicateurs & Angle Isovist & Coeff. & $\begin{array}{l}\text { Log- } \\
\text { Vraisemblance }\end{array}$ \\
\hline 21. Ecart-type des longueurs de rayon & $360^{\circ}$ & $-2,38 * * *$ & $-14,24$ \\
16. Jaggedness & $104^{\circ}$ & $1,61^{* *}$ & $-11,36$ \\
\hline Log-Vrais. initiale -25,56 & $* * p<0,05$ & $* * * p<0,01$ & \\
Log-Vrais. finale $-11,36$ & & & \\
Pseudo- $R^{2}$ ajusté $\quad 0,48$ & & & \\
& & &
\end{tabular}

Le modèle associé aux passants est légèrement moins performant que celui des habitants. Même si la différence est peu significative, cela signifie que leurs comportements de mobilité sont moins prédictibles. Cette remarque est intéressante si l'on rappelle que la longueur des itinéraires empruntés est également plus importante dans cette catégorie enquêtée. Il y a donc, sans surprise, chez les passants, une rationalité moindre qui s'exprime à la fois sur l'effort physique de déplacement et sur l'effort cognitif associé aux ambiances. La valeur prise par le pseudo- $\mathrm{R}^{2}$ ajusté est cependant suffisamment élevée pour nous permettre d'analyser les résultats pris par le modèle. Il semble ainsi que les passants cherchent à réduire 
la complexité de l'espace visible puisque l'écart-type des longueurs de rayons est négatif. Les formes complexes sont évitées par les passants ce qui facilite sans doute la lisibilité et l'appréhension des espaces traversés. Le Jaggedness, qui complète le modèle de la catégorie des passants, traduit l'attrait pour des espaces dont le périmètre présente un caractère assez allongé ou dentelé. A priori, cela peut sembler contradictoire avec l'écart-type des longueurs de rayons. Mais cela peut aussi signifier qu'ils sont attirés par des espaces caractérisés par une perspective visuelle qui leur facilite l'orientation. Cet attrait pour des perspectives visuelles confirme des hypothèses déjà largement exprimées par les spécialistes de la syntaxe spatiale et du mouvement naturel (par exemple Hillier et Hanson, 1984). Il semble donc y avoir un compromis entre forme homogène et perspectives visuelles allongées. De ce point de vue, il serait sans doute utile d'examiner la succession des valeurs prises par ces indices le long des itinéraires traversés par les passants. L'utilisation d'indices entropiques au cours du cheminement pourrait constituer une méthode d'agrégation intéressante des valeurs morphométriques mesurées dans les différents points composant les itinéraires étudiés. Il conviendrait également d'explorer spatialement la répartition des valeurs prises par les différentes variables car cela influe nécessairement sur les résultats obtenus. Par son fonctionnement, le modèle logit permet d'éviter un biais important des méthodes agrégées : si un ensemble de choix présente des caractéristiques homogènes pour une variable morphologique donnée, celle-ci ne pourra être sélectionnée car elle ne permettra pas de discriminer significativement le trajet emprunté. En revanche, rien ne garantit que cette variable est perçue de manière neutre par l'enquêté. Certaines variables non retenues dans les modèles ci-dessus ne sont donc pas nécessairement non pertinentes.

La méthode devra être reproduite et les résultats validés dans d'autres espaces urbains et avec un échantillon d'enquêtés plus conséquent. Mais, globalement, l'ensemble de ces modèles propose des résultats intéressants dans la mesure où ils mettent en évidence un lien entre morphologie et comportements de mobilité à un niveau désagrégé.

\section{Conclusion}

En aménagement urbain, la valorisation de l'espace est un objectif recherché. Mais comment concevoir un environnement urbain perçu de manière positive par la majorité des citadins ? Au-delà de l'environnement construit, c'est l'espace habité et pratiqué qui doit focaliser l'attention des aménageurs. Dans cette optique, il faut mobiliser des méthodes basées sur le relevé de pratiques individuelles au regard des espaces sensibles. A cet effet, la modélisation des choix individuels de fréquentation des espaces conduit à établir, par exemple, un lien entre des indicateurs mesurables, objectifs et des ambiances privilégiées du point de vue des usages même si une part non négligeable des comportements n'est pas prévisible (Besse, 2010). Le piéton est en effet un capteur d'ambiances imparfait : il ne voit pas tout et n'est pas capable de faire une synthèse parfaite de ce qu'il perçoit. Mais ses actes sont tout de même de bons révélateurs de ses préférences et, à ce titre, riches d'enseignements. 
Aucune des deux méthodes utilisées, le calcul d'indices morphométriques et l'utilisation d'un modèle de choix discret, n'est en soit innovante mais leur couplage est très intéressant. L'apport des indicateurs morphométriques, classiquement utilisés en architecture et en planification urbaine, comme variables d'entrée pour alimenter la compréhension des choix d'itinéraires semble pertinent. Quant aux modèles de choix, ils constituent un outil facilement mobilisable pour faire le lien entre environnement construit et comportements de mobilité. En somme, la démarche présentée a avant tout une portée heuristique. Elle permet de questionner les relations entre formes urbaines construites et expérience sensorielle des individus (perceptions, préférences, usages...). Dans cet article, la modélisation des pratiques de mobilité a permis de mettre en évidence certaines caractéristiques morphologiques attractives pour les piétons. Si la méthode semble prometteuse, il convient toutefois de rester prudent et il n'est pas question ici de vouloir généraliser les résultats obtenus avec un si faible échantillon. Par ailleurs, la démarche présente l'avantage d'être assez facilement reproductible et «instrumentable »: les jeux de données et les méthodes utilisés peuvent être mis en œuvre sur d'autres terrains d'études et à plus grande échelle. Les premiers résultats obtenus par la méthode présentée ouvrent la voie à des travaux intégrant d'autres indicateurs morphométriques, notamment ceux, plus réalistes, basés sur une approche en trois dimensions. Il serait aussi souhaitable d'intégrer des aspects relatifs aux densités perçues ainsi que des éléments plus fonctionnels. La confrontation à des préférences déclarées par les individus pourrait également constituer une piste d'enrichissement de la méthode et de validation des résultats ${ }^{15}$.

Par ailleurs, le modèle logit multinomial utilisé peut être remplacé par d'autres modèles de choix. En effet, dans un environnement complexe se pose la question de la difficulté de la prise de décision des individus. La rationalité renvoie à la capacité à choisir l'option la plus satisfaisante et aux moyens d'atteindre ce but. Comme le rappellent les psychologues, la rationalité est toujours limitée, a fortiori quand le choix porte sur des caractéristiques spatiales par nature difficiles à évaluer, ce qui est notamment le cas de la morphologie des espaces urbains. Or, si les individus se livrent à une comparaison très précise des alternatives quand le contexte de choix est simple, ces derniers utilisent des règles de décision simplifiées dès lors que la prise de décision devient complexe. Selon Kahneman et Tversky (1979), pour appréhender ces situations de choix complexes et diminuer la difficulté de la tâche de décision, les individus peuvent recourir à des « heuristiques » c'est-à-dire à des raisonnements volontairement simplifiés. Ainsi, certains modèles de choix séquentiels, non compensatoires, ont été proposés dans lesquels seule une partie de l'information est considérée. Enfin, signalons également que la prise de décision repose sur deux attitudes contradictoires : l'effort consacré à la prise de décision et la qualité de la décision prise. Le choix peut donc être appréhendé comme le résultat d'un compromis entre l'effort cognitif mis en œuvre (dépendant de la complexité du

15 . Comme mentionné en partie 3, des éléments de discours ont été recueillis lors de l'enquête mobilité. L'analyse discursive, que l'on ne peut à proprement parler assimiler à des préférences déclarées, a cependant permis de révéler certaines concordances intéressantes avec les préférences révélées par les modèles de choix. 
choix, du temps disponible pour choisir, des capacités du décideur) et la précision des résultats obtenus (Payne et al., 1993). Il semble donc essentiel de proposer une modélisation des choix davantage adaptée à la complexité des ambiances urbaines abordée ici par le biais d'indicateurs morphométriques.

\section{Remerciements}

Ce travail a été effectué dans le cadre du Programme Interdisciplinaire de Recherche Ville et Environnement (CNRS-MEDDLT). 


\section{Bibliographie}

Alfonzo M.A. (2005). To walk or not to walk? The hierarchy of walking needs. Environment and Behavior, vol. $37, \mathrm{n}^{\circ}$ 6, p. 808-836.

Amar G. (1993). Pour une écologie urbaine des transports. Les annales de la recherche urbaine, $\mathrm{n}^{\circ}$ 59-60, p. 140-151.

Augoyard J.F. (1979). Pas à pas, essai sur le cheminement quotidien en milieu urbain, Ed. du Seuil, Paris.

Bel Hadj Ali A. (2001). Qualité géométrique des entités géographiques surfaciques: application à l'appariement et définition d'une typologie des écarts géométriques. Thèse en Sciences de l'Information Géographique, Université de Marne-la-Vallée.

Benedikt M.L. (1979). To take hold of space: isovists and isovist fields. Environment and Planning B: Planning and Design, vol. 6, $\mathrm{n}^{\circ}$ 1, p. 47-65.

Besse J.-M. (2010). Le paysage, espace sensible, espace public. META: Research in Hermeneutics, Phenomenology, and Practical Philosophy, vol. 2, n ${ }^{\circ}$. p. 259-286.

Bierlaire M. (1998). Discrete choice models. Operations research and decision aid methodologies in traffic and transportation management. Heidelberg, Springer-Verlag, p. 203-227.

Breheny M. J. (1992). Sustainable Development and Urban Form, Pion, London.

Cervero R., Kockelman K. (1997). Travel demand and the 3Ds: density, diversity, and design, Transportation Research Part D, vol. 2, n 3, p. 199-219.

Conroy Dalton R., Dalton N. (2001). OmniVista: an application for isovist field and path analysis, 3rd International Space Syntax Symposium, Atlanta, Georgia, USA.

Costermans J. (2001). Les activités cognitives. Raisonnement, décision et résolution de problèmes, Ed. De Boeck Université, Bruxelles.

Couclelis H. (1992). People manipulate objects (but cultivate fields): Beyond the raster-vector debate in GIS. Theories and Methods of Spatio-Temporal Reasoning in Geographic Space, International Conference GIS - From Space to Territory: Theories and Methods of Spatio-Temporal Reasoning. Pisa, Italy, Springer, p. 65-77.

de Palma A., Thisse J.F. (1989). Les modèles de choix discrets. Annales d'Economie et de Statistique, $\mathrm{n}^{\circ}$ 9, p. 151-190.

de Palma A., Meyer G.M., Papageorgiou Y.Y. (1994). Rational choice under an imperfect ability to choose. American Economic Review, vol. 84, n 3 , p. 419-440.

Ettema D., Arentze T., Timmermans H.J.P. (2011). Social influences on household location, mobility and activity choice in integrated micro-simulation models. Transportation Research Part A: Policy and Practice, vol. 45, n 4, 283-295.

Foltête J.C., Piombini A. (2010). Deviations in pedestrian itineraries in urban areas: a method to assess the role of environmental factors. Environment and Planning B: Planning and Design, vol. $37, \mathrm{n}^{\circ}$ 4, p. 723-739.

Franz G., Wiener J.M. (2005). Exploring isovist-based correlates of spatial behavior and experience, Proceedings of the 5th International Space Syntax Symposium, Delft. 
Gärling T., Friman M. (2002). A psychological approach to the study of residential choice and satisfaction. Residential Environments: Choice, Satisfaction, and Behavior. London, Bergin \& Garvey, p. 55-80.

Golledge R. (1999). Human wayfinding and cognitive maps. Wayfinding behaviour, Cognitive mapping and other spatial processes. Baltimore, John Hopkins University Press, p. 5-45.

Hensher D.A. (1994). Stated preference analysis of travel choices: the state of practice. Transportation, vol. $21, \mathrm{n}^{\circ} 2$, p. 107-133.

Hillier B., Hanson J. (1984). The Social Logic of Space, Cambridge, Cambridge University press.

Kahneman D., Tversky A. (1979). Prospect theory: An analysis of decision under risk. Econometrica, vol. 27, n $^{\circ}$ 2, p. 263-292.

Leduc T., Tourre V., Woloszyn P., Miguet F. (2011). Measuring surrounding space to assess the pedestrian visual aperture angle in the urban fabric: towards a kurtosis based isovist indicator. Information fusion and geographic information systems: Towards the digital ocean. Berlin, Heidelberg, Springer-Verlag, p. 129-141.

Lee D.R., Sallee G.T. (1970). A Method of Measuring Shape. Geographical Review, vol. 60, $\mathrm{n}^{\circ} 4$, p. $555-563$.

Loiteron D., Bishop I. (2005). Simulating visitor movement: autonomous agents in urban public garden, CUPUM '05, Londres.

Louviere J.J., Hensher D., Swait J. (2000). Stated choice methods: Analysis and applications in marketing, transportation and environmental valuation, Cambridge University Press, Cambridge.

Lynch K. (1969). L'image de la cité, Dunod, Paris.

MacEachren A.M. (1985). Compactness of geographic shape: comparison and evaluation of measures. Geografiska Annaler, vol. 67, n 1, p. 53-67.

Maignant G. (2005). Compacité et forme urbaine, une analyse environnementale dans la perspective d'un développement urbain durable. Actes du colloque Développement urbain durable, gestion des ressources et gouvernance, Lausanne, Switzerland.

McFadden D. (1981). Econometric models of probabilistic choice. Structural analysis of discrete data with econometric applications. Cambridge, MIT Press, p. 198-272.

Miller V.C. (1953). A quantitative geomorphic study of drainage basin characteristics in the clinch mountain area Virginia and Tennessee. Project NR 389-042, Tech. Report 3. Columbia University, Department of Geology, New York.

Panerai P., Demorgon M., Depaule J.-C. (1999). Analyse urbaine, Parenthèses, Marseille.

Payne J., Bettman J., Johnson E. (1993). The adaptive decision maker, Cambridge Univ. Press, New York.

Peneau J.P. (2000). Les ambiances urbaines. Données urbaines 3. Paris, Anthropos, p. $375-$ 386.

Peponis J., Wineman J., Rashid M., Hong Kim S., Bafna S. (1997). On the description of shape and spatial configuration inside buildings: convex partitions and their local properties. Environment and Planning B: Planning and Design, vol. 24, n ${ }^{\circ}$, p. 761-781. 
31 Géomatique. Volume $-\mathrm{n}^{\circ}$

Prato C.G. (2009). Route choice modelling: past, present and future research directions. Journal of choice modelling, vol. 2, ${ }^{\circ} 1$, p. 65-100.

Ratti C. (2004). Space syntax: some inconsistencies. Environment and Planning B: Planning and Design, vol. 31, $\mathrm{n}^{\circ} 4$, p. 487-499.

Santos M. (1997). La nature de l'espace: technique et temps, raison et émotion, L'Harmattan, Paris.

Sarradin F. (2004). Analyse morphologique des espaces ouverts urbains le long de parcours. Thèse en Sciences pour l'Ingénieur, Université de Nantes.

Shriver K. (1997). Influence of environmental design on pedestrian travel behaviour in four Austin neighborhoods. Transportation Research Record, vol. 1578, p. 64-75.

Stamps III A.E. (2002). Entropy, visual diversity, and preference. The Journal of general psychology, vol. 129, $\mathrm{n}^{\circ}$ 3, p. 300-20.

Stamps III A.E. (2005). Isovists, enclosure, and permeability theory. Environment and Planning B: Planning and Design, vol. 32, $\mathrm{n}^{\circ}$ 5, p. 735-762.

Stamps III A.E. (2010). Slines, entropy, and environmental exploration. Environment and Planning B: Planning and Design, vol. 37, $\mathrm{n}^{\circ}$ 4, p. 704-722.

Teller J. (2003). A spherical metric for the field-oriented analysis of complex urban open spaces. Environment and Planning B: Planning and Design, vol. 30, n ${ }^{\circ}$, p. 339-356.

Thibault G., Mari J.L., Sequeira J. (2007). Indices de formes: de la 2D à la 3D. Journées du Laboratoire des Sciences de l'Information et des Systèmes, vol. 1, p. 1-11.

Thibaud J.-P. (2002). L'horizon des ambiances urbaines. Communications, vol. 73. p. 185201.

Thomas R. (2004). Quand le pas fait corps et sens avec l'espace. Aspects sensibles et expressifs de la marche en ville, http://cybergeo.revues.org/4304.

Turner A., Dox, M., O’Sullivan D., Penn A. (2001). From isovists to visibility graphs: a methodology for the analysis of architectural space. Environment and Planning B: Planning and Design, vol. 28, $\mathrm{n}^{\circ}$ 1, p. 103-121.

Woloszyn P., Leduc, T. (2011). A landscape potential characterization: spatial template of pedestrian ambient fields within the urban fabric, IT-Gatineau, lère conference intercontinentale en Intelligence Territoriale. Gatineau, Canada. http://hal.archivesouvertes.fr/docs/00/63/40/58/PDF/itgatineau2011-woloszynLeduc.pdf

Zacharias J. (1997). The impact of layout and visual stimuli on the itineraries and perception of pedestrians in a public market. Environment and Planning $B$, vol. $24, \mathrm{n}^{\circ} 1$, p. 23-35.

Zacharias J. (2006). Exploratory spatial behaviour in real and virtual environments. Landscape and Urban Planning, vol. 78, $\mathrm{n}^{\circ} 1-2, \mathrm{p} .1-13$. 